\title{
The effect of weak inertia on the emptying of a tube
}

\author{
Alain de Ryck ${ }^{\text {a) }}$ \\ École des Mines d'Albi-Carmaux, route de Teillet, 81013 Albi Cedex 09, France
}

(Received 26 October 2000; accepted 1 April 2002; published 21 May 2002)

\begin{abstract}
We present an extension of the classical axisymmetric Bretherton theory giving the thickness of the liquid film left on the walls of a drained tube, treating the case of weak inertia by a regular perturbation method. The results obtained by numerical integration fit Taylor's [J. Fluid Mech. 10, 161 (1961)] experiments, obtained with viscous fluids (glycerine and strong sucrose solutions), and Aussillous and Quéré's [Phys. Fluids 12, 2367 (2000)] experiments with low viscosity liquids (hexamethyldisiloxane and water) when inertia becomes important. The discrepancies observed between the theory and high Reynolds numbers experiments $(\mathrm{Re}>1000)$ are commented on. (C) 2002 American Institute of Physics. [DOI: 10.1063/1.1480267]
\end{abstract}

\section{INTRODUCTION}

Coating flows, which are liquid flows leading to a deposited liquid layer on the surface of a solid, have been extensively studied ${ }^{1}$ for their practical importance in many technologies: Painting, printing, emulsion deposition in photographic industry, air displacement in wetted porous media. This last topic has received some attention for its relevance to oil recovery and to the understanding of air penetration into the lungs. An idealized situation, sketched in Fig. 1, is the emptying of a pore or a capillary filled with a wetting liquid and drained by pushing a fluid of lower viscosity, leading to a deposited liquid layer. The experimental work by Taylor, ${ }^{2}$ measuring the amount of several highly viscous liquids left behind when the liquid is blown out of a tube, showed a limit in liquid recovery when increasing the drainage velocity. The thickness $e$ of the deposited film has a limiting value of approximately one third.

Bretherton $^{3}$ studied, both experimentally and theoretically, the drainage of a capillary at low velocity. His theory, valid when the deposited film is thin compared to the tube radius, also describes the two-dimensional (2D) drainage between two parallel plates, and is similar to the one proposed by Landau and Levich ${ }^{4}$ for plate or wire coating. In all these cases, the velocity dependence of the deposited film thickness is written

$$
e=1.34 \kappa^{-1} \mathrm{Ca}^{2 / 3}
$$

where $\mathrm{Ca}=\eta V / \gamma$ is the capillary number, which compares the viscous and capillary forces ( $\eta$ and $\gamma$ are, respectively, the viscosity and surface tension of the liquid and $V$ is the drainage velocity). $\kappa$ is the difference of curvature between the static meniscus (weakly deformed at low velocity) and the thin film. For a tube of radius $r$, a Hele-Shaw cell of spacing $r$, or a fiber of radius $r$, we have $\kappa^{-1}=r$. For a plate withdrawn out of a liquid bath, $\kappa^{-1}=\sqrt{\gamma / 2 \rho g}$, where $\rho$ is

${ }^{a)}$ Electronic mail: deryck@enstimac.fr the specific density of the fluid. The numerical constant is obtained by matching the curvature of the meniscus with the asymptotic curvature of the thin film. ${ }^{3,4}$

At higher velocity, some discrepancies are observed between Eq. (1) and experimental results. For plate coating, the drainage by gravity of the film becomes non-negligible. For the tube (respectively wire) coating, a first semiempirical correction, proposed by White and Tallmadge for wire coating, ${ }^{5}$ is to write $\kappa^{-1}=r-e$ (respectively, $r+e$ ). This extends the validity of the theoretical Bretherton law to situations in which the thickness of the remaining film becomes comparable with the capillary radius. In the tube case, a limiting value at high capillary numbers is then found: $e / r$ $\rightarrow 1$, quite different from Taylor's observations: $:^{2} e / r \rightarrow 0.34$.

To go to higher velocities, $\operatorname{Cox}^{6}$ proposed a theory giving the amount of liquid left on the walls of the tube based on an exponential shape for the driving bubble but the fit with the Taylor's results is not so good. The two-dimensional finite element calculation with a free interface by Reinelt and Saffman, ${ }^{7}$ leads to a very good agreement with these experimental results, but does not take into account inertia. But, for liquids of low viscosity like water or ethanol, encountered in washing processes for example, the liquid inertia leads to a noticeable thickening of the remaining film, for drainage at capillary numbers smaller than unity. ${ }^{8}$

Giavedoni and Saita ${ }^{9}$ presented numerical results, both in the axisymmetric and plane cases, obtained by a boundary integral method. ${ }^{10}$ Their work included the inertial forces and they found a thinning effect due to inertia for Reynolds numbers up to 70. This work has been recently completed by Heil ${ }^{11}$ in the $2 \mathrm{D}$-channel case using a finite element method. It demonstrates a small thickening effect due to inertia for higher Reynolds numbers up to 280 .

Here, we propose an extension of the Bretherton's classical axisymmetric analysis to include weak fluid inertia effects. This study is an alternative of the Giavedoni and Saita ${ }^{9}$ and Heil ${ }^{11}$ works. Its numerical part is reduced to the resolution of a third-order ordinary differential equation, ${ }^{12,13}$ and is inspired by previous theoretical work introducing weak fluid inertia in plate ${ }^{14-16}$ and wire coating, ${ }^{17,18}$ and by $2 \mathrm{D}$ 


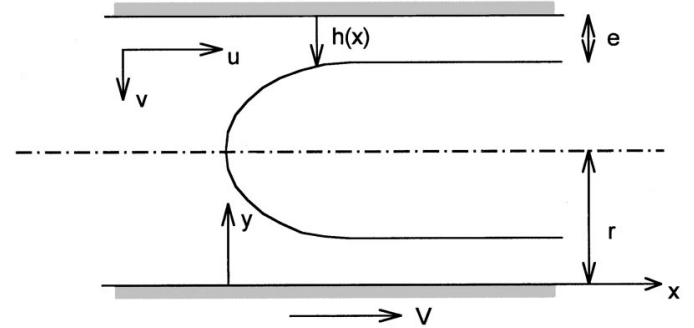

FIG. 1. Sketch of a tube full of liquid and drained by an air bubble.

channel drainage. ${ }^{19}$ In the latter case, the theory takes also into account the drainage by gravity.

After the presentation of the equations describing the drainage of a cylinder in Sec. II, they are solved at leading order in Sec. III using the lubrication approximation. In Sec. IV, a first-order approximation is used, introducing the inertial terms. Two cases are then studied: Highly viscous liquids and liquids of low viscosity. The results obtained are compared, respectively, with the Taylor's experiments ${ }^{2}$ and the recent ones by Aussillous and Quéré. ${ }^{8}$

\section{DESCRIPTION OF THE PROBLEM}

We consider an infinite tube of circular geometry (radius $r$ ), full of liquid and drained by air at a constant velocity $V$. In a frame attached to the air bubble, we define the axial origin and direction $x$ from the apex to the air side. The radial direction $y$ is chosen from the tube wall inwards. In these coordinates, the axial and radial liquid velocities are $u$ and $v$. In this frame, the walls of the tube have velocity $V$. The profile of the film surrounding the bubble is $h(x)$, decreasing from $r$ to the constant value $e$ for $x$ ranging from 0 to infinity.

The steady-state Navier-Stokes equations in these coordinates are written ${ }^{3}$

$\eta\left\{\frac{\partial^{2} u}{\partial x^{2}}+\frac{\partial^{2} u}{\partial y^{2}}+\frac{1}{r-y} \frac{\partial u}{\partial r}\right\}=\frac{\partial p}{\partial x}+\rho\left(v \frac{\partial u}{\partial y}+u \frac{\partial u}{\partial x}\right)$

and

$$
\begin{gathered}
\eta\left\{\frac{\partial^{2} v}{\partial x^{2}}+\frac{\partial^{2} v}{\partial y^{2}}-\frac{1}{r-y} \frac{\partial v}{\partial y}-\frac{1}{(r-y)^{2}} v\right\} \\
=\frac{\partial p}{\partial y}+\rho\left(u \frac{\partial v}{\partial y}+u \frac{\partial v}{\partial x}\right)
\end{gathered}
$$

where $\eta$ and $\rho$ are, respectively, the viscosity and specific mass of the liquid, and $p$ the pressure. The liquid is incompressible, so we have

$$
\frac{\partial v}{\partial y}-\frac{v}{r-y}+\frac{\partial u}{\partial x}=0 .
$$

The boundary conditions at the walls are

$$
\text { for } y=0, \quad u=V \quad \text { and } v=0 .
$$

On the air-liquid interface, $y=h(x)$, neglecting the gas viscosity, the stresses are due to the interface curvature and are written

$$
p_{s} \underline{n}-\underline{\sigma}_{s} \underline{n}=p_{o} \underline{n}-\gamma c \underline{n},
$$

where $\gamma$ is the surface tension of the liquid and $p_{o}$ the air pressure. The subscript $s$ means that the value is taken at the liquid-gas interface. The vector $\underline{n}$ is normal to the surface and $\underline{\underline{\sigma}}$ the viscous stress tensor. They are written

$$
\underline{n}=\frac{1}{\sqrt{1+h_{x}^{2}}}\left(\begin{array}{c}
1 \\
-h_{x}
\end{array}\right)
$$

and

$$
\underline{\underline{\sigma}}=\eta\left(\begin{array}{cc}
2 \frac{\partial v}{\partial y} & -\frac{\partial u}{\partial y}-\frac{\partial v}{\partial x} \\
-\frac{\partial u}{\partial y}-\frac{\partial v}{\partial x} & 2 \frac{\partial u}{\partial x}
\end{array}\right),
$$

where the subscript $x$ means differentiation with respect to the axial coordinate. The curvature $c$ is given by

$$
c=\frac{h_{x x}}{\left(1+h_{x}^{2}\right)^{3 / 2}}+\frac{1}{(r-h) \sqrt{1+h_{x}^{2}}} .
$$

\section{LEADING ORDER SOLUTION}

Assuming the slope of the thin film to be small and radial velocity to be much smaller than the axial velocity, the radial component of the Navier-Stokes equation (3) reduces to $\partial p / \partial y=0$. The pressure is then uniform in the thin film and is written, using the radial projection of Eq. (4)

$$
p=p_{s}=p_{o}-\gamma c, \quad \text { with } \quad c=h_{x x}+\frac{1}{r-h} .
$$

Equation (6), in the axial direction yields

$$
\left.\frac{\partial u}{\partial y}\right|_{s}=0 .
$$

Finally, Eq. (2), the axial component of the Navier-Stokes equation, reduces to

$$
\frac{\partial}{\partial y}\left((r-y) \frac{\partial u}{\partial y}\right)=\frac{1}{\eta} p_{x}(r-y),
$$

may be integrated twice to give the velocity profile

$$
u=V+\frac{1}{\eta} p_{x}\left(\frac{(r-y)^{2}}{4}-\frac{r^{2}}{4}-\frac{(r-h)^{2}}{2} \ln \frac{r-y}{r}\right) .
$$

A last integration gives the flux $Q$, which is a constant in steady-state regime. Its value is related to the thickness $e$ of the deposited film by

$$
Q=\int_{h}^{r} 2 \pi y u d y=\int_{e}^{r} 2 \pi y V d y .
$$

Introducing Eq. (12) into Eq. (13) leads to 


$$
\begin{aligned}
(r-h)^{2}-(r-e)^{2} & \\
= & \frac{1}{\mathrm{Ca}} c_{x}\left(\frac{r^{4}}{8}-\frac{r^{2}(r-h)^{2}}{2}+\frac{3}{8}(r-h)^{4}\right. \\
& \left.+\frac{(r-h)^{4}}{2} \ln \frac{r-h}{r}\right) .
\end{aligned}
$$

Finally, since the normalized thickness $E=e / r$, ratio between the thickness of the deposited film and the tube radius, remains a small parameter, we have truncated Eq. (14) to the two first terms of its Taylor expansion close to $E=0$. It then reduces to

$$
Y_{X X X}=3 \frac{1-Y}{Y^{3}}\left(1+E \frac{Y-1}{2}\right)-\frac{E^{2}}{\mathrm{Ca}^{2 / 3}} Y_{X},
$$

when using the same reduced variables as those used by Bretherton: ${ }^{3}$ A film thickness scaled by $e$, the thickness of the film at infinity $(h=e Y)$, and an axial coordinate scaled by a length $\ell$, where $\ell=e \mathrm{Ca}^{-1 / 3}(x=\ell X)$.

We then obtain a generalized version of the Landau-Levich ${ }^{3,4}$ equation with the additional terms in $E$ coming from the cylindrical geometry: The first from the expression of the flux, the second from the radial curvature.

\section{A. Numerical integration}

Since the profile does not depend explicitly on $x$, we use the thickness $y$ as the variable of integration and integrate numerically a second order differential equation for $G(Y)$ $=Y_{X}$. This method leads to more stability in the numerical integration (we have used a commercial code-ode45 routine from Matlab-based on a 4 to 5 Runge-Kutta method ${ }^{20}$ ), but does not allow to find a nonmonotonous profile since by construction, we impose a univocal relation between $y$ and its derivative $y_{x}$.

The integration is started close to the thin film using the linearized conditions: ${ }^{3,13} \quad Y_{o}=1+e^{s X}, \quad Y_{o}^{\prime}=s e^{s X}$ and $Y_{o}^{\prime \prime}$ $=s^{2} e^{s X}$, where $\epsilon=e^{s X}$ is a small quantity $\left(10^{-4}\right.$ in our calculations) and where $s$ is solution of the following equation obtained by linearization of Eq. (15):

$$
s^{3}+\frac{E^{2}}{\mathrm{Ca}^{2 / 3}} s+3=0 .
$$

The initial conditions for $G$ then is written

$$
G(1+\epsilon)=s \epsilon, \quad \text { and } \frac{d G}{d Y}(1+\epsilon)=s .
$$

For a given $\mathrm{Ca}$ and $E$, we integrate $G$ from $Y=1+\epsilon$ to $1 / E$. Figure 2(a) shows several bubble profiles obtained for $\mathrm{Ca}$ $=0.05$.

We observe that there is a critical thickness $y$ at which a matching to a sphere is possible, i.e., there exists a point on the profile (small circles in Fig. 2) where the two radii of curvature are equal

$$
Y_{X X}=\frac{E}{\mathrm{Ca}^{2 / 3}} \frac{1}{1-E Y} \text {. }
$$
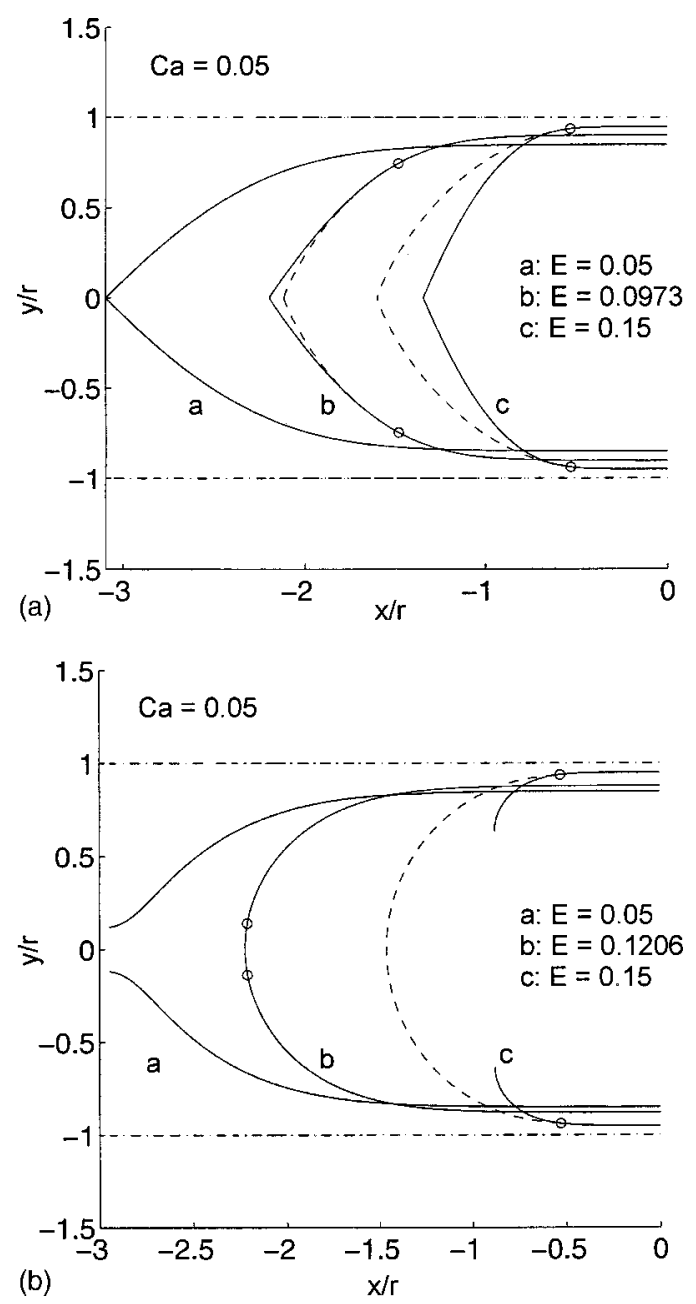

FIG. 2. (a) Approximate curvature. Bubble profile for $\mathrm{Ca}=0.05$ and $E$ $=0.05,0.0973$, and 0.15. Dash and dotted lines: Walls of the tube. Dashed lines: approximated spheres $\left(y_{x x}=-1 /(r-y)\right)$. Circles: matching points; there is no matching for $E>0.0973$. (b) Exact curvature. Bubble profile for $\mathrm{Ca}=0.05$ and $E=0.05,0.1206$, and 0.15 .

With this matching, we insure that the profile ends with a static zone where the pressure is constant. This matching is represented in Fig. 2(a). The profiles are matched at the circle point with a curve solution of $h_{z z}=-1 /(r-h)$ (dotted lines). $E=0.0973$ is the highest value allowing a matching at $\mathrm{Ca}=0.05$. There is no matching feasible for $E=0.05$ for this value of the capillary number.

\section{B. Results}

In Fig. 3, we have compared this maximum matching thickness (dotted line) with the experimental values obtained by Taylor (denoted by squares). In the same figure, these results are also compared to the asymptotic matching by Bretherton [Eq. (1), dashed and dotted line]. Both are only valid for very small capillary numbers, but the denominator in Eq. (18) ensures a saturation effect.

In order to improve the accuracy, we used the full expression of the curvature. The film profile is then given by

$$
C_{X}=3 \frac{1-Y}{Y^{3}}\left(1+E \frac{Y-1}{2}\right),
$$




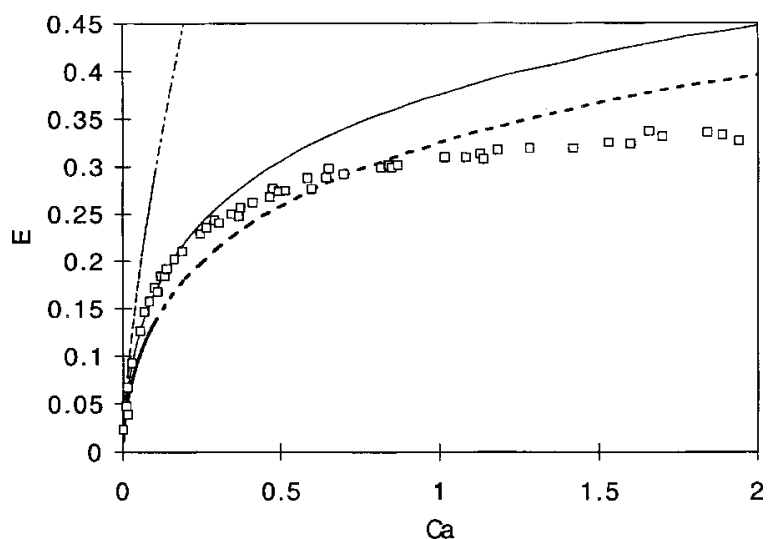

FIG. 3. Thickness of the deposited film scaled by $r$ vs the capillary number. Dashed and dotted line: Eq. (1). Dashed line: Maximum thickness allowing a matching with a spherical cap using an approximated curvature. Solid line: the same, but using an exact curvature expression. Squares: Taylor's experiments (Ref. 2).

where $C$ is the normalized curvature

$$
C=\frac{Y_{X X}}{\left(1+\mathrm{Ca}^{2 / 3} Y_{X}^{2}\right)^{3 / 2}}+\frac{E}{\mathrm{Ca}^{2 / 3}} \frac{1}{(1-E Y)\left(1+\mathrm{Ca}^{2 / 3} Y_{X}^{2}\right)^{1 / 2}} .
$$

With this correction, we obtain more realistic bubble profiles, as shown in Fig. 2(b). As previously, for each capillary number, a maximum thickness allowing matching is found. This value fits the experimental data well for capillary numbers up to 0.25 as can be observed in Fig. 3 (solid line). Nevertheless, we still observe some discrepancies for higher capillary numbers which here do not come from inertia since, in the Taylor's experiments, the Reynolds numbers $(\operatorname{Re}=\rho V r / \eta)$ were always lower than unity.

\section{FIRST-ORDER SOLUTION}

To achieve a more accurate solution when the thickness of the deposited film increases, we need to obtain the firstorder correction the leading order result described in the previous section. Up to the first order in $\mathrm{Ca}^{1 / 3}$, the terms previously neglected have been written using the expressions of the velocity obtained at the zeroth order.

To first order, the pressure at the liquid-bubble interface is written

$$
p_{s}=-\gamma c+\left.2 \eta \frac{\partial v_{o}}{\partial y}\right|_{s},
$$

where the subscript $o$ means that the velocity is computed at the zeroth order. Using the incompressibility of the liquid [Eq. (4)] leads to

$$
p_{s}=-\gamma c-\left.2 \eta \frac{\partial u_{o}}{\partial x}\right|_{s}-2 \eta \frac{u_{o s}}{h} h_{x},
$$

where $u_{o}$ is given by Eqs. (12) and (13)

$$
\begin{aligned}
u_{o}= & V\left(1+8 \frac{(r-h)^{2}-(r-e)^{2}}{r^{4}-4 r^{2}(r-h)^{2}+3(r-h)^{4}-4(r-h)^{4} \ln (r-h) / r}\right. \\
& \left.\times\left(\frac{(r-y)^{2}}{2}-\frac{r^{2}}{2}-\frac{(r-h)^{2}}{2} \ln \frac{r-y}{r}\right)\right) .
\end{aligned}
$$

The pressure profile inside the thin film is given by Eq. (3) at first order:

$$
\eta\left\{\frac{\partial}{\partial y}\left(-\frac{\partial u_{o}}{\partial x}\right)\right\}=\frac{\partial p}{\partial y},
$$

and leads together with Eq. (22) to

$$
p=-\gamma c-\left.\eta \frac{\partial u_{o}}{\partial x}\right|_{s}-\eta \frac{\partial u_{o}}{\partial x}+2 \eta \frac{u_{o s}}{r-h} h_{x} .
$$

At this same order, Eq. (2) is written

$$
\eta\left\{\frac{1}{r-y} \frac{\partial}{\partial y}\left((r-y) \frac{\partial w}{\partial y}\right)\right\}=\frac{\partial p}{\partial x}+\rho\left(v_{o} \frac{\partial u_{o}}{\partial y}+u_{o} \frac{\partial u_{o}}{\partial x}\right) .
$$

The last step is to integrate this equation three times in order to obtain the flux as in the previous section and then to expand the expression versus $E$. We obtain finally in the reduced Landau-Bretherton coordinates

$$
\begin{aligned}
C_{X}= & 3 \frac{1-Y}{Y^{3}}\left(1+E \frac{Y-1}{2}\right)+3 \frac{\mathrm{Ca}^{2 / 3}}{Y^{3}}\left\{Y _ { X X } Y \left[\frac{43-7 Y}{40}\right.\right. \\
& \left.+E \frac{-129+270 Y-61 Y^{2}}{240}\right] \\
& \left.+Y_{X}^{2}\left[\frac{-41-2 Y}{20}+E \frac{41-39 Y-2 Y^{2}}{40}\right]\right\} \\
& +\frac{1}{35} F E \mathrm{Ca}^{4 / 3} \frac{Y_{X}}{Y^{3}}\left[6\left(-9+Y+Y^{2}\right)\right. \\
& \left.+E \frac{1728-1131 Y-171 Y^{2}+134 Y^{3}}{32}\right],
\end{aligned}
$$

where $C$ is still the reduced curvature given by Eq. (20). $F$ $=\rho \gamma r / \eta^{2}=\mathrm{Re} / \mathrm{Ca}$ is a nondimensional number which depends only on the nature of the liquid and of the radius of the tube. For water in a tube of radius $1 \mathrm{~mm}, F=72000$. For glycerol in the same tube, $F=0.36$.

In Eq. (27), two new terms appears, compared to Eq. (19), both expanded in powers of $E$. The first one is a correction to the lubrication approximation. After Bretherton, Spiers et al. ${ }^{21}$ introduced this correction in the case of plate coating. The second one is an inertial correction. First introduced by Esmail and Hummel ${ }^{14}$ for the plate coating problem, this inertial term has been shown to lead to a thickening of the film entrained by a plate ${ }^{16}$ or a fiber withdrawn from of a liquid bath. ${ }^{18}$

Together with Eq. (20), Eq. (27) leads again to a third ordinary differential equation for the film profile. The numerical integration still starts from a point close to the thin film: $Y=1+e^{s X}$, where $s$ is obtained by the linearization of the system. With the new terms, Eq. (16) is replaced by 


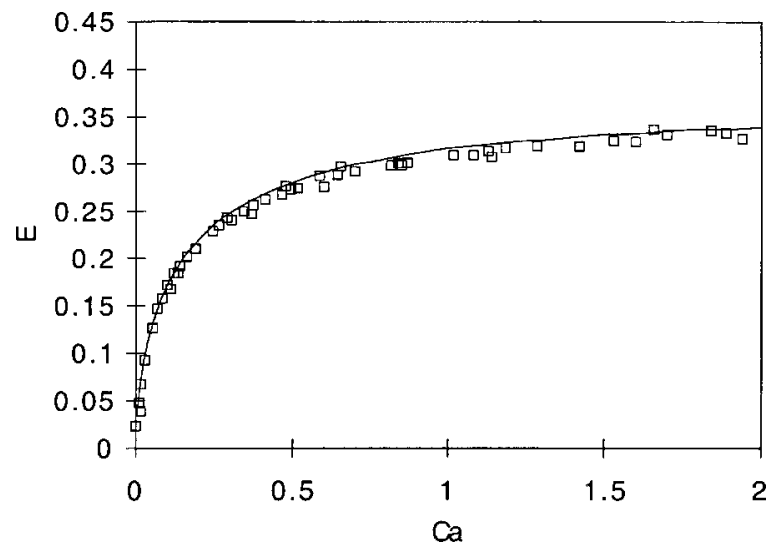

FIG. 4. Taylor's experiments (Ref. 2) and the maximum value of $E$ allowing a bubble profile ending like a sphere for $F=0$.

$$
\begin{aligned}
s^{3}+ & \frac{E^{2}}{\mathrm{Ca}^{2 / 3}} \frac{s}{(1-E)^{2}}+3-\mathrm{Ca}^{2 / 3} s^{2}\left(\frac{27}{10}+E\right) \\
& +F E \mathrm{Ca}^{4 / 3} s\left(\frac{6}{5}-\frac{E}{2}\right)=0 .
\end{aligned}
$$

The final step is the same as previously. For a given parameter number $F$, we search, for each value of the capillary number $\mathrm{Ca}$, the maximum value of the reduced film thickness $E$ allowing a matching of the film profile with a spherical cap. This has been done in two cases. First, with $F=0$, for liquids of high viscosity, and then for parameter numbers higher than 10000 , representative of liquids of low viscosity.

\section{A. Highly viscous liquids}

For highly viscous liquids, the inertial correction remains negligible for capillary number less than unity, as is the case of the experiments by Taylor. ${ }^{2}$ Figure 4 compares them with the maximum thickness allowing a bubble profile ending with a spherical cap with $F=0$. The agreement is quite good. In Fig. 5, several bubble shapes obtained for $\mathrm{Ca}$ ranging from 0.001 to 1 are compared. The matching points,

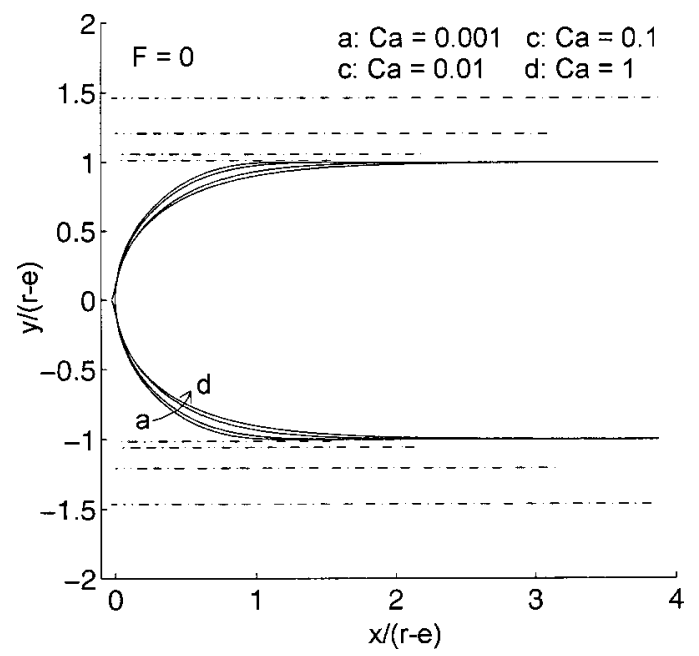

FIG. 5. From left to right: bubble shapes for $\mathrm{Ca}=0.001 ; 0.01 ; 0.1$, and 1 . Dash and dotted lines: Positions of the walls.
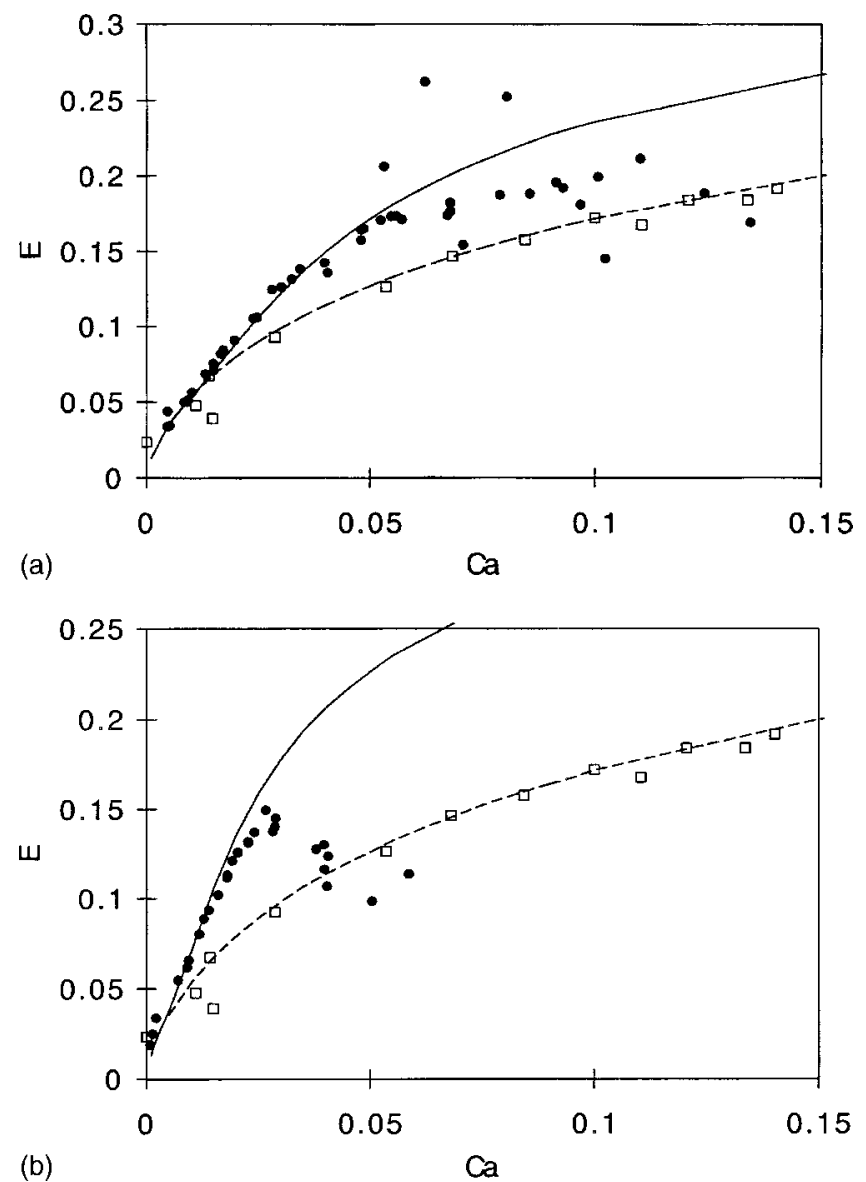

FIG. 6. (a) Experimental deposited film thicknesses and the theoretical maximum ones allowing a matching with a spherical cap. Black points: Experiments with hexamethyldisiloxane (Ref. 8) $\left(\rho=0.76 \mathrm{~kg} / \mathrm{m}^{3}, \eta=0.5 \mathrm{cP}\right.$ and $\gamma=15.9 \mathrm{mN} / \mathrm{m})$ and a tube of radius $0.4 \mathrm{~mm}(F=19330)$. Solid line: Theory with the same parameter. White points: Taylor's experiments (Ref. 2). Dashed line: Theory with $F=0$. (b) Experimental deposited film thicknesses and the theoretical maximum ones allowing a matching with a spherical cap. Black points: Experiments with hexamethyldisiloxane (Ref. 8) and a tube of radius $1.4 \mathrm{~mm}(F=67670)$. Solid line: Theory with the same parameter. White points: Taylor's experiments (Ref. 2). Dashed line: Theory with $F=0$

not shown, are close to the axis of revolution except for $\mathrm{Ca}=1$. In that latter case, the matching occurs at half the bubble size. In all cases we observe that the bubbles are only slightly deformed: the thin film of constant thickness is attained above a distance $x$ from the top of the cap close to $(r-e)$.

\section{B. For liquids of low viscosity}

Finally, the system of equations has been integrated using as parameter $F=19330$ and 67670 , values representative of a low viscosity silicone oil drained out of a tube of radius 0.4 and $1.4 \mathrm{~mm}$. The maximum thickness curves are compared in Fig. 6 with the Aussillous and Quéré experiments. $^{8}$

We observe that the threshold and the beginning of thickening due to inertia are well represented by the theory. Nevertheless, for higher capillary numbers, the theory overestimates the experimental values obtained. Several hypotheses may be put forward to explain this discrepancy. First, 


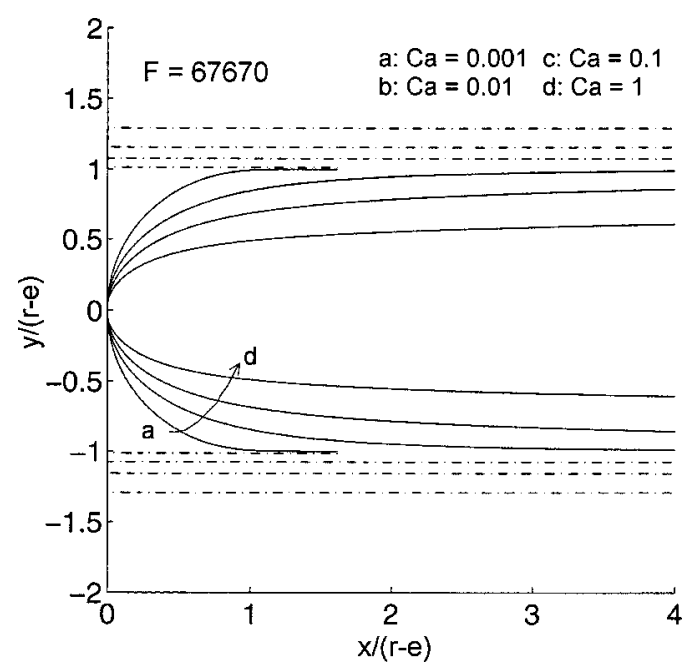

FIG. 7. From left to right: bubble shapes for $F=67670$ and $\mathrm{Ca}=0.001$, $0.01,0.1$, and 1 .

the theory presented here includes inertia as a first order perturbation and therefore has no reason to describe high inertia situations. Secondly, the method used computes only steady-state axisymmetric bubble profiles, with no oscillations of the film thickness and of maximum thickness for a given capillary number. This solution is perhaps not the most stable at high velocity. In particular, further work should be made to check if the maximum thickness criterion leads, at high velocity, to the less dissipative solution.

Finally, there are some limitations to this visco-inertial regime in the Aussillous and Quéré's experiments. ${ }^{8}$ As described by the authors, the finite length of the liquid reservoir leads to a deposited film thickness limited by the viscous boundary layer. Another point is that the steady-state viscoinertial regime is not reached in these experiments, which were performed with an air-liquid front displacement of about $20 \mathrm{~cm}$. But it can be observed in the simulations that the size of the transition zone between the apex and the thin

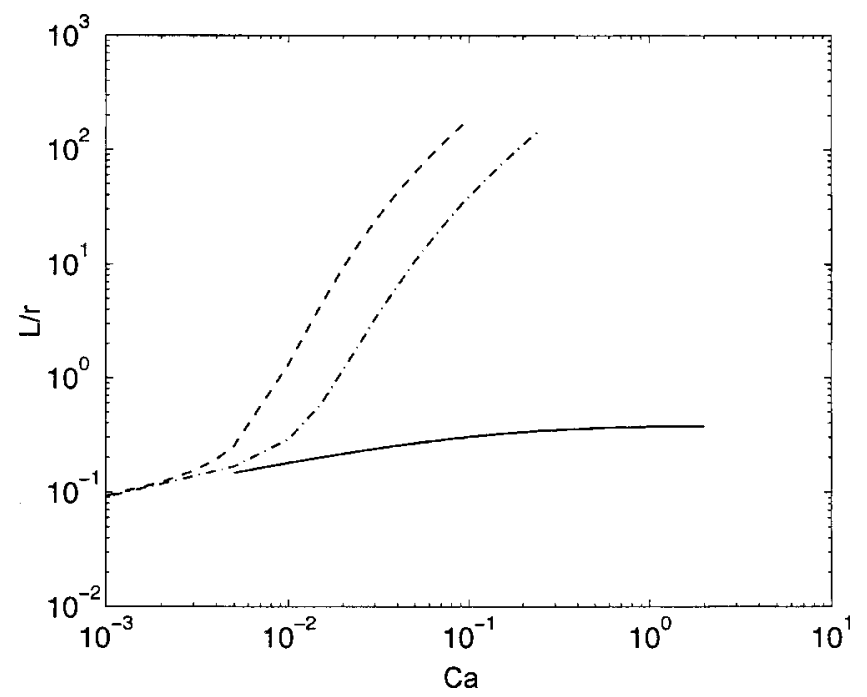

FIG. 8. Size of the transition zone scaled by $r$ vs Ca. Black line: $F=0$. Dashed and dotted line: $F=19330$. Dashed line: $F=67670$.

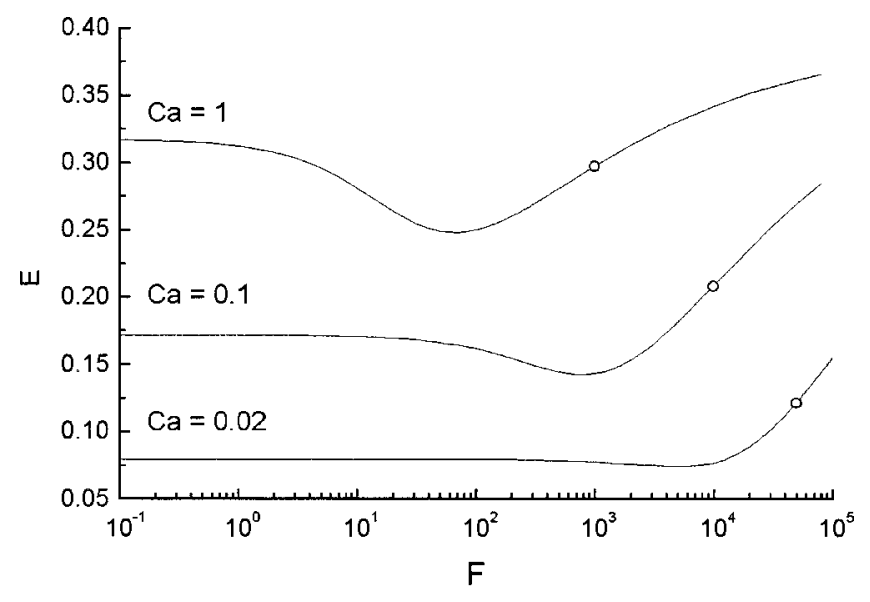

FIG. 9. Maximum deposited thickness (scaled by $r$ ) $E$ vs $F$ for three capillary numbers. The circles indicates $\mathrm{Re}=1000$.

film, given by $L=\ell / s$ becomes very large compared to the tube radius $r$ at high Reynolds numbers. As an example, we have plotted on Fig. 7 the profiles obtained for $F=67670$. We observe that, contrary to the $F=0$ case (Fig. 5), the bubble is highly deformed for $\mathrm{Ca} \geqslant 0.01$. In Fig. 8, we have plotted this length $L$ of the transition zone, scaled by $r$, versus the capillary number. We observe that as long as inertia remains negligible, the bubble length is small compared to its width. But when inertia becomes important $(\operatorname{Re}>1000)$, the bubble length grows and may reach about $10 \mathrm{~cm}$.

\section{CONCLUDING REMARKS}

We presented an extension of the classical axisymmetric Bretherton theory, including weak inertial effects, based on a regular perturbation method. By numerical integration of the film profile and looking for the maximum value of the deposited film thickness allowing a matching with a spherical cap, a velocity dependence of this thickness is obtained and, for liquids of low viscosity, a thickening due to inertia is observed. The agreement between these numerical results and the experimental results up to moderate Reynolds numbers $(\operatorname{Re}<1000)$ is quite good. This thickening is not what is reported by Giavedoni and Saita. ${ }^{9}$ They report a slight thinning due to inertia for Reynolds numbers up to 70 . We indeed retrieve this result when looking at low Reynolds or $F$ numbers, as shown on Fig. 9 where $E$, the film thickness scaled by $r$, is plotted versus the number $F$ for different capillary numbers. In particular, it can be noticed that this thinning, which may attain $20 \%$ for $\mathrm{Ca}=1$, is maximum for Re around 100. This could explain why the last experimental points by Taylor are slightly under the theoretical curve in Fig. 4. The values of $F$ for the experiments he performed at a capillary number greater than one may be evaluated at around 0.5 (glycerol and tube radius of $1.5 \mathrm{~mm}$ ). In Fig. 9, it can be seen that this leads to a correction of $6 \%$ for $\mathrm{Ca}=1$.

Finally, some points remain to be investigated. The thickness numerically obtained is a maximum thickness and it is not clear why it is this thickness which gives good correspondence with the experimental values. 
Another remaining issue is the description of the rapid draining of capillaries, for Reynolds numbers higher than 1000. For the present problem, a finite Reynolds numbers theory, like the numerical theory by Heil, ${ }^{11}$ is necessary.

\section{ACKNOWLEDGMENTS}

It is a pleasure to thank Pascale Aussillous and David Quéré for fruitful discussions.

${ }^{1}$ K. J. Ruschak, "Coating flows," Annu. Rev. Fluid Mech. 17, 65 (1985).

${ }^{2}$ G. I. Taylor, "Deposition of a viscous fluid on the wall of a tube," J. Fluid Mech. 10, 161 (1961).

${ }^{3}$ F. P. Bretherton, "The motion of long bubbles in tubes," J. Fluid Mech. 10, 166 (1961)

${ }^{4}$ L. D. Landau and B. Levich, "Dragging of a liquid by a moving plate," Acta Physicochim. USSR 17, 42 (1942); also in V. G. Levich, Physical Hydrodynamics (Prentice-Hall, Englewood Cliffs, 1962).

${ }^{5}$ D. A. White and J. A. Tallmadge, "A theory of withdrawal of cylinders from liquid baths," AIChE J. 12, 333 (1966).

${ }^{6}$ B. G. Cox, "On driving a viscous fluid out of a tube," J. Fluid Mech. 14, 81 (1962).

${ }^{7}$ D. A. Reinelt and P. G. Saffman, "The penetration of a finger into a viscous fluid in a channel and tube," SIAM (Soc. Ind. Appl. Math.) J. Sci. Stat. Comput. 6, 542 (1985).

${ }^{8} \mathrm{P}$. Aussillous and D. Quéré, "Quick deposition of a fluid on the wall of a tube," Phys. Fluids 12, 2367 (2000).

${ }^{9}$ M. D. Giavedoni and F. A. Saita, "The axisymmetric and plane cases of gas phase steadily displacing a Newtonian liquid-A simultaneous solution of the governing equations," Phys. Fluids 9, 2420 (1997).

${ }^{10}$ M. J. Martinez and K. S. Udell, "Axisymmetric creeping motion of drops through circular tubes,” J. Fluid Mech. 210, 565 (1990).

${ }^{11}$ M. Heil, "Finite Reynolds number effects in the Bretherton problem," Phys. Fluids 13, 2517 (2001)

${ }^{12}$ B. G. Higgins, W. J. Silliman, R. A. Brown, and L. E. Scriven, "Theory of meniscus shape in film flows. A synthesis," Ind. Eng. Chem. Fundam. 16, 393 (1977).

${ }^{13}$ E. O. Tuck and L. W. Schwartz, "A numerical and asymptotic study of some third-order ordinary differential equations relevant to draining and coating flows," SIAM Rev. 32, 453 (1990).

${ }^{14}$ A. J. Soroka and J. A. Tallmadge, "A test of the inertial theory for plate withdrawal," AIChE J. 17, 505 (1971).

${ }^{15}$ M. N. Esmail and R. L. Hummel, "Nonlinear theory of free coating onto a vertical surface," AIChE J. 21, 958 (1975).

${ }^{16}$ A. de Ryck and D. Quéré, "Gravity and inertia effects in plate coating," J. Colloid Interface Sci. 203, 278 (1998).

${ }^{17}$ A. Koulago, D. Quéré, A. de Ryck, and V. Shkadov, "Film entrained by a fiber quickly drawn out of a liquid bath," Phys. Fluids 7, 1221 (1995).

${ }^{18}$ A. de Ryck and D. Quéré, "Inertial coating of a fibre," J. Fluid Mech. 311, 219 (1996).

${ }^{19}$ G. C. Gardner and G. A. Adebiyi, "The liquid film left behind a large bubble in a sloping channel," Chem. Eng. Sci. 29, 461 (1974).

${ }^{20}$ J. R. Dormand and P. J. Prince, "A family of embedded Runge-Kutta formulae,” J. Comput. Appl. Math. 6, 19 (1980).

${ }^{21}$ R. P. Spiers, C. V. Subbaraman, and W. L. Wilkinson, "Free coating of a Newtonian liquid onto a vertical surface," Chem. Eng. Sci. 29, 389 (1974). 